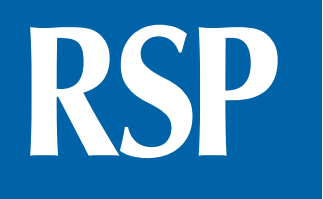

http://www.rsp.fsp.usp.br/
Revista de Saúde Pública

\title{
Óbitos e internações decorrentes de intoxicações por medicamentos com prescrição e isentos de prescrição, no Brasil
}

\author{
Fernanda Gross Duarte' iD, Marcelo Neubauer de Paula" iD, Nelzair Araújo Vianna"l' iD, Maria \\ Conceição Chagas de Almeida"I' (iD, Edson Duarte Moreira Junior ${ }^{\text {III }}$ (iD) \\ ' Pós-Graduação em Biotecnologia em Saúde e Medicina Investigativa. Instituto Gonçalo Moniz, Fundação \\ Oswaldo Cruz. Salvador, BA, Brasil \\ " Grupo Hypera Pharma. São Paulo, SP, Brasil \\ III Fundação Oswaldo Cruz. Instituto Gonçalo Moniz. Laboratório de Epidemiologia Molecular e Bioestatística. \\ Salvador, BA, Brasil
}

\section{RESUMO}

OBJETIVO: Estimar a incidência de hospitalizações por intoxicação medicamentosa e a mortalidade desses agravos no Brasil, descrevendo as tendências de 2009 a 2018.

MÉTODOS: Os dados de internações hospitalares e óbitos originam-se do DATASUS e os dados demográficos, do Instituto Brasileiro de Geografia e Estatística (IBGE). Foram selecionadas as internações hospitalares cuja Autorização para Internação Hospitalar (AIH) indicasse como procedimento "tratamento de intoxicação ou envenenamento por exposição a medicamento e substâncias de uso não medicamentoso", sendo analisados apenas os casos de hospitalização por intoxicação medicamentosa. A incidência de hospitalização e a mortalidade foram calculadas separadamente para intoxicações causadas por medicamentos com prescrição (MRx) e isentos de prescrição (MIP).As taxas foram ainda estratificadas por sexo, faixa etária e região de residência no Brasil. A análise de tendência foi realizada por regressão linear generalizada pelo método de Prais-Winsten.

Correspondência:

Rua Waldemar Falcão, 121 40296-710 Candeal, Salvador, BA

E-mail: nandagross@gmail.com

Recebido: 31 jan 2021

Aprovado: 6 abr 2021

Como citar: Duarte FG, Paula MN, Vianna NA, Almeida MCC, Moreira Jr ED. Óbitos e internações decorrentes de intoxicações por medicamentos com prescrição e isentos de prescrição, no Brasil. Rev Saude Publica. 2021;55:81. https://doi.org/10.11606/s15188787.2021055003551

Copyright: Este é um artigo de acesso aberto distribuído sob os termos da Licença de Atribuição Creative Commons, que permite uso irrestrito, distribuição e reprodução em qualquer meio, desde que o autor e a fonte originais sejam creditados.
RESULTADOS: A maioria das internações foi causada por MRx (97\%), com mortalidade aproximadamente 50 vezes maior, quando comparada às internações por MIP. A tendência da incidência das internações por MRx foi estacionária, mas a mortalidade aumentou durante o período estudado, enquanto a tendência na mortalidade e na incidência de internações por MIP foi decrescente.

CONCLUSÕES: As internações por intoxicação medicamentosa, sobretudo aquelas causadas por MRx, têm grande impacto e importância na saúde pública, especialmente porque esses agravos podem ser prevenidos.

DESCRITORES: Medicamentos sem Prescrição, envenenamento. Medicamentos sob Prescrição, envenenamento. Hospitalização. Envenenamento, mortalidade. 


\section{INTRODUÇÃO}

Intoxicação medicamentosa é decorrente da exposição a um medicamento em doses superiores àquelas usualmente empregadas para profilaxia, diagnóstico, tratamento ou para modificação de funções fisiológicas, podendo resultar em diferentes sinais e sintomas, a depender do tipo de medicamento e do metabolismo do fármaco. . A intoxicação pode ocorrer de forma não intencional ou acidental, quando resulta de automedicação, de erro na dosagem, de terapêutica inadequada, de troca de medicamentos ou de ingestão involuntária; ou intencional, quando relacionada ao abuso, ao uso indevido ou à autolesão ${ }^{2}$. Os casos graves de intoxicação, levando a internações hospitalares e até mesmo ao óbito, constituem-se num importante problema de saúde pública ${ }^{3}$.

Entre 2009 e 2018, o Sistema Nacional de Informações Tóxico-Farmacológicas (Sinitox) registrou 254.135 casos de intoxicação no Brasil, com um total de 710 óbitos (0,28\%). Os medicamentos foram a principal causa de intoxicação dentre todos os agentes notificados, cerca de $29 \%$ dos casos no período, seguidos por animais peçonhentos, produtos de limpeza domésticos e agrotóxicos ${ }^{4}$. Desde 1994, os fármacos ocupam o primeiro lugar no ranking de intoxicações e o segundo lugar em número de óbitos. Em 2016, segundo dados do Sinitox, a intoxicação acidental foi uma das principais causas das ocorrências com medicamentos, representando cerca de um terço dos casos notificados $(32,7 \%)^{4}$.

Na maioria dos países desenvolvidos, a situação é semelhante à do Brasil, com medicamentos liderando as causas de intoxicações exógenas 5 . Nos Estados Unidos da América (EUA), dados nacionais de 2018 indicam que mais de dois milhões de casos de intoxicação foram causados por medicamentos ${ }^{6}$. Além disso, observou-se uma tendência crescente das taxas de mortalidade por intoxicações medicamentosas entre os anos de 1994 e 2010, em todas as regiões e grupos demográficos nos EUA, sendo a intoxicação acidental a principal causa $(71 \%)^{7}$.

No Brasil, os medicamentos podem ser enquadrados como medicamentos de prescrição (MRx) ou medicamentos isentos de prescrição (MIP). Essa classificação foi estabelecida pela primeira vez na legislação sanitária brasileira, em 1973, quando se regulamentou o controle sanitário do comércio de medicamentos. Nessa ocasião, inseriu-se o conceito de medicamento de venda livre como aquele que independe de prescrição, estabelecendo-se diferenças quanto à rotulagem e à publicidade dos produtos prescritos ${ }^{8}$. Em 2016, a Agência Nacional de Vigilância Sanitária (Anvisa) definiu mais claramente os critérios para que um medicamento seja classificado como "isento de prescrição", sendo considerados nessa categoria medicamentos de 33 grupos terapêuticos, com as devidas exceções ${ }^{9}$. Os critérios para a inclusão de medicamentos na lista de MIP levam em conta aspectos como: tempo de comercialização, segurança do medicamento, sintomas identificáveis, utilização por curto período de tempo, ser manejável pelo paciente, apresentar baixo potencial de risco e não apresentar dependência9.

O objetivo deste estudo foi estimar a incidência de hospitalizações por intoxicação medicamentosa, no geral e de acordo com o tipo de medicamento (com prescrição ou isento), bem como a mortalidade desses agravos no Brasil, descrevendo as tendências observadas nessas estimativas no período entre 2009 e 2018.

\section{MÉTODOS}

Esse é um estudo retrospectivo para determinar as taxas de internações hospitalares e óbitos, causados por intoxicação medicamentosa no Brasil. Realizou-se uma revisão dos dados sobre intoxicações medicamentosasna base do Sistema Único de Saúde (Datasus), num período de 10 anos (data da saída em: 2009 a 2018), incluindo informações como: sexo, idade, local de residência e tipo de intoxicação medicamentosa. Os resultados 
referentes às intoxicações causadas por medicamentos com prescrição são apresentados separadamente daqueles causados por medicamentos sem prescrição.

Os dados de internações e óbitos são do portal do DATASUS e foram extraídos pelo software TabWin, fornecido pelo DATASUS. Os dados demográficos foram obtidos do Censo $2010 \mathrm{e}$ das estimativas intercensitárias para os demais anos, disponibilizados pelo portal de dados do Instituto Brasileiro de Geografia e Estatística (IBGE) ${ }^{10}$.

Inicialmente, foram selecionadas as internações hospitalares informadas entre $2009 \mathrm{e}$ 2018, que a Autorização para Internação Hospitalar (AIH) solicitava como procedimento "Tratamento de intoxicação ou envenenamento por exposição a medicamento e substâncias de uso não medicamentoso". Posteriormente, foram mantidas apenas as internações nas quais esse procedimento era confirmado, sendo excluídas as internações consideradas para outra finalidade no relatório de alta/óbito. Os casos de intoxicação medicamentosa foram classificados, conforme os grupos terapêuticos, como causados por medicação com prescrição ou isenta, utilizando-se as informações do CID primário e secundário. Neste artigo foram analisados os casos decorrentes de intoxicação medicamentosa.

A incidência de hospitalização foi calculada dividindo-se o número de casos de internação ocorridos na população de estudo pelo número de habitantes correspondente ao período e local analisado. De maneira análoga, as taxas de mortalidade foram calculadas dividindo-se o número de óbitos pela população total em cada período do estudo. Uma vez que as taxas brutas podem ser influenciadas pela estrutura etária das populações de diferentes regiões e em períodos de tempo diferentes, as taxas estimadas foram padronizadas por faixa etária pelo método direto, utilizando-se, como padrão, a população sugerida pela Organização Mundial de Saúde (OMS 2000-2025), permitindo, assim, analisar tendências e fazer comparações desses dados ${ }^{11}$. A análise das frequências, taxas de hospitalizações e dos óbitos foi realizada segundo tipo de intoxicação medicamentosa classificada como: causada por medicamentos com prescrição (MRx) ou por medicamentos isentos de prescrição (MIP). As taxas foram estratificadas por sexo, faixa etária e região de residência no Brasil. A análise de tendência da série histórica foi realizada por regressão linear generalizada pelo método de Prais-Winste ${ }^{12}$, com correção para o efeito de autocorrelação de primeira ordem $^{13}$. A tendência de internações hospitalares/óbitos foi considerada estacionária quando $\mathrm{p}>0,05$; declinante quando $\mathrm{p}<0,05$ e coeficiente da regressão negativo; ou ascendente quando $\mathrm{p}<0,05$ e coeficiente da regressão positivo ${ }^{14}$. As análises estatísticas foram realizadas utilizando-se o programa estatístico Stata (Stata Statistical Software: Release 16. College Station, TX: StataCorp LLC).

\section{RESULTADOS}

No Brasil, ocorreram 85.811 internações hospitalares devido à intoxicação medicamentosa entre 2009 e 2018, sendo 97\% delas causadas por intoxicação por MRx e apenas $3 \%$ causadas por MIP. A incidência média de internações por MRx (4,16 por 100 mil habitantes) foi muito superior à por MIP (0,13 por 100 mil habitantes), RR = 32,8 (IC95\% 28,9-37,1). Houve 2.644 óbitos $(3,08 \%)$ entre as hospitalizações no período do estudo. A taxa de mortalidade em casos decorrentes de intoxicação por MRx $(3,11 \%)$ foi maior que a dos óbitos por intoxicação por MIP (1,93\%).

A Tabela 1 mostra a frequência das internações hospitalares e dos óbitos causados por intoxicação medicamentosa segundo região e outras características sociodemográficas. A maioria das internações ocorreu no sexo feminino tanto nas intoxicações por MRx (56,7\%) como naquelas por MIP $(54,9 \%)$. A incidência média de hospitalizações por MRx também foi maior no sexo feminino (4,61 por 100 mil) comparado ao masculino (3,67 por 100 mil), mas essa diferença foi menor nas internações por MIP. Contrariamente, o sexo feminino representou apenas um quarto dos óbitos nas internações por MIP e pouco menos da metade daqueles nas internações por MRx. A cor da pele/raça branca foi a mais 
comumente reportada nas internações por MRx e MIP, em quase um terço dos casos não havia informação sobre a cor da pele. A região sudeste teve o maior número de internações por MRx, seguida da região sul e da centro-oeste. A distribuição regional das internações por MIP foi semelhante (Tabela 1).

A incidência de hospitalização e a mortalidade segundo faixa etária são apresentadas na Tabela 2. As intoxicações, tanto por MRx como por MIP, tiveram maior incidência de internações em menores de 5 anos, diminuindo a frequência conforme o aumento da idade,

Tabela 1. Frequência de hospitalizações e óbitos por tipo de intoxicação medicamentosa, Brasil, 2009 a 2018.

\begin{tabular}{|c|c|c|c|c|c|c|c|c|c|c|c|c|}
\hline & \multicolumn{4}{|c|}{ Medicamentos com prescrição (MRx) } & \multicolumn{4}{|c|}{ Medicamentos isentos de prescrição (MIP) } & \multicolumn{4}{|c|}{ Total } \\
\hline & \multicolumn{2}{|c|}{ Hospitalizações } & \multicolumn{2}{|c|}{ Óbitos } & \multicolumn{2}{|c|}{ Hospitalizações } & \multicolumn{2}{|c|}{ Óbitos } & \multicolumn{2}{|c|}{ Hospitalizações } & \multicolumn{2}{|c|}{ Óbitos } \\
\hline & $\mathbf{n}$ & $(\%)$ & $\mathbf{n}$ & $\%$ & $\mathbf{n}$ & $\%$ & $\mathbf{n}$ & $\%$ & $\mathbf{n}$ & $\%$ & $\mathbf{n}$ & $\%$ \\
\hline Total & 83.275 & 100 & 2.595 & 100 & 2.536 & 100 & 49 & 100 & 85.811 & 100 & 2.644 & 100 \\
\hline \multicolumn{13}{|l|}{ Sexo } \\
\hline Feminino & 47.252 & 56,7 & 1.267 & 48,8 & 1.391 & 54,9 & 13 & 26,5 & 48.643 & 56,7 & 1.280 & 48,4 \\
\hline Masculino & 36.023 & 43,3 & 1.328 & 51,2 & 1.145 & 45,1 & 36 & 73,5 & 37.168 & 43,3 & 1.364 & 51,6 \\
\hline \multicolumn{13}{|l|}{ Faixa etária } \\
\hline$<5$ & 7.600 & 9,1 & 40 & 1,5 & 529 & 20,9 & 4 & 8,2 & 8.129 & 9,5 & 44 & 1,7 \\
\hline $5-9$ & 3.399 & 4,1 & 14 & 0,5 & 194 & 7,6 & 1 & 2,0 & 3.593 & 4,2 & 15 & 0,6 \\
\hline $10-14$ & 4.623 & 5,6 & 29 & 1,1 & 144 & 5,7 & 0 & 0 & 4.767 & 5,6 & 29 & 1,1 \\
\hline $15-19$ & 8.468 & 10,2 & 129 & 5,0 & 279 & 11,0 & 2 & 4,1 & 8.747 & 10,2 & 131 & 5,0 \\
\hline 20-29 & 15.679 & 18,8 & 401 & 15,5 & 476 & 18,8 & 7 & 14,3 & 16.155 & 18,8 & 408 & 15,4 \\
\hline $30-39$ & 14.851 & 17,8 & 504 & 19,4 & 304 & 12,0 & 10 & 20,4 & 15.155 & 17,7 & 514 & 19,4 \\
\hline $40-49$ & 11.857 & 14,2 & 543 & 20,9 & 257 & 10,1 & 4 & 8,2 & 12.114 & 14,1 & 547 & 20,7 \\
\hline $50-59$ & 7.497 & 9,0 & 401 & 15,5 & 155 & 6,1 & 7 & 14,3 & 7.652 & 8,9 & 408 & 15,4 \\
\hline $60-69$ & 4.331 & 5,2 & 244 & 9,4 & 88 & 3,5 & 6 & 12,2 & 4.419 & 5,1 & 250 & 9,5 \\
\hline 70 ou mais & 4.970 & 6,0 & 290 & 11,2 & 110 & 4,3 & 8 & 16,3 & 5.080 & 5,9 & 298 & 11,3 \\
\hline \multicolumn{13}{|l|}{ Cor da pele/raça } \\
\hline Branca & 32.814 & 39,4 & 921 & 36 & 911 & 35,9 & 11 & 22,4 & 33.725 & 39,3 & 932 & 35,2 \\
\hline Parda & 22.076 & 26,5 & 704 & 27 & 768 & 30,3 & 14 & 28,6 & 22.844 & 26,6 & 718 & 27,2 \\
\hline Preta & 2.787 & 3,3 & 93 & 4 & 77 & 3,0 & 3 & 6,1 & 2.864 & 3,3 & 96 & 3,6 \\
\hline Outra & 835 & 1,1 & 25 & 1 & 24 & 1,0 & 0 & 0 & 859 & 1,1 & 25 & 1,0 \\
\hline Não informada & 24.763 & 29,7 & 852 & 33 & 756 & 29,8 & 21 & 42,9 & 25.519 & 29,7 & 873 & 33,0 \\
\hline \multicolumn{13}{|l|}{ Regiões } \\
\hline Norte & 3.868 & 4,6 & 108 & 4,2 & 142 & 5,6 & 5 & 10,2 & 4.010 & 4,7 & 113 & 4,3 \\
\hline Nordeste & 7.064 & 8,5 & 172 & 6,6 & 317 & 12,5 & 5 & 10,2 & 7.381 & 8,6 & 177 & 6,7 \\
\hline Centro-Oeste & 14.375 & 17,3 & 621 & 23,9 & 545 & 21,5 & 20 & 40,8 & 14.920 & 17,4 & 641 & 24,2 \\
\hline Sudeste & 41.522 & 49,9 & 1278 & 49,2 & 977 & 38,5 & 11 & 22,4 & 42.499 & 49,5 & 1.289 & 48,8 \\
\hline Sul & 16.446 & 19,7 & 416 & 16,0 & 555 & 21,9 & 8 & 16,3 & 17.001 & 19,8 & 424 & 16,0 \\
\hline \multicolumn{13}{|c|}{ Caráter da internação } \\
\hline Urgência & 81.513 & 97,9 & 2.536 & 97,7 & 2.474 & 97,6 & 49 & 100 & 83.987 & 97,9 & 2.585 & 97,8 \\
\hline Eletivo & 1.762 & 2,1 & 59 & 2,3 & 62 & 2,4 & 0 & 0 & 1.824 & 2,1 & 59 & 2,2 \\
\hline
\end{tabular}

Incidência de hospitalizações por MRx: 4,61 por 100 mil (em mulheres) e 3,67 por 100 mil (em homens). Incidência de hospitalizações por MIP: 0,14 por 100 mil (em mulheres) e 0,12 por 100 mil (em homens).

Mortalidade por MRx: 123 por milhão (em mulheres) e 135 por milhão (em homens).

Mortalidade por MIP: 1,3 por milhão (em mulheres) e 3,7 por milhão (em homens). 
sobretudo nas hospitalizações por MIP. A média da mortalidade no período estudado foi maior nas internações por MRx do que por MIP, RR = 52,0 (IC95\% 21,5-126,0). Indivíduos com 60 anos ou mais tiveram a maior taxa de mortalidade nas intoxicações por MRx e MIP, seguido pelo grupo menor de 5 anos nas internações por MIP.

A Tabela 3 apresenta a incidência de hospitalização e a mortalidade, padronizadas para a distribuição da população mundial (OMS 2000-2025), segundo as regiões do país. No período do estudo, a maior incidência média de internações por MRx ocorreu na região sul e sudeste, enquanto as regiões norte e nordeste apresentaram as menores taxas. Nas hospitalizações por MIP, a incidência média foi maior na região centro-oeste e sul. De forma semelhante, as regiões norte e nordeste apresentaram as menores taxas. A mortalidade observada nas intoxicações por MRx foi mais elevada nas regiões sudeste e sul, enquanto as causadas por MIP teve maior taxa na região nordeste.

O número total de hospitalizações e óbitos, os coeficientes de regressão e a tendência de hospitalizações e de mortalidade por tipo de intoxicação medicamentosa, segundo região

Tabela 2. Incidência de hospitalização e mortalidade por intoxicação medicamentosa segundo faixa etária, Brasil, 2009-2018.

\begin{tabular}{|c|c|c|c|c|c|c|c|c|c|c|c|}
\hline & \multicolumn{10}{|c|}{ Ano } & \multirow{2}{*}{$\begin{array}{l}\text { Média } \\
\text { anual }\end{array}$} \\
\hline & 2009 & 2010 & 2011 & 2012 & 2013 & 2014 & 2015 & 2016 & 2017 & 2018 & \\
\hline \multicolumn{12}{|l|}{ Hospitalização ${ }^{\mathrm{a}}$} \\
\hline \multicolumn{12}{|l|}{ MRx (faixa etária) } \\
\hline 0 a 4 anos & 7,62 & 6,10 & 5,37 & 5,99 & 5,57 & 5,67 & 4,88 & 5,07 & 5,27 & 5,67 & 5,72 \\
\hline 5 a 19 anos & 4,47 & 3,23 & 3,32 & 3,25 & 3,25 & 2,95 & 2,86 & 2,83 & 3,85 & 4,40 & 3,44 \\
\hline 20 a 59 anos & 7,00 & 4,58 & 4,40 & 4,49 & 4,32 & 3,90 & 3,66 & 3,62 & 3,99 & 4,45 & 4,44 \\
\hline 60 anos ou mais & 4,49 & 3,55 & 3,37 & 3,09 & 3,23 & 3,45 & 3,44 & 3,26 & 3,66 & 3,51 & 3,50 \\
\hline Total & 6,10 & 4,22 & 4,07 & 4,11 & 4,00 & 3,73 & 3,52 & 3,48 & 3,99 & 4,37 & 4,16 \\
\hline \multicolumn{12}{|l|}{ MIP (faixa etária) } \\
\hline 0 a 4 anos & 0,58 & 0,27 & 0,42 & 0,42 & 0,49 & 0,41 & 0,34 & 0,32 & 0,43 & 0,30 & 0,40 \\
\hline 5 a 19 anos & 0,15 & 0,11 & 0,08 & 0,13 & 0,13 & 0,14 & 0,15 & 0,13 & 0,13 & 0,14 & 0,13 \\
\hline 20 a 59 anos & 0,20 & 0,11 & 0,12 & 0,10 & 0,11 & 0,09 & 0,09 & 0,08 & 0,08 & 0,08 & 0,11 \\
\hline 60 anos ou mais & 0,14 & 0,08 & 0,10 & 0,10 & 0,05 & 0,08 & 0,08 & 0,06 & 0,04 & 0,04 & 0,08 \\
\hline Total & 0,21 & 0,12 & 0,13 & 0,13 & 0,13 & 0,12 & 0,12 & 0,11 & 0,10 & 0,10 & 0,13 \\
\hline \multicolumn{12}{|l|}{ Mortalidade $^{b}$} \\
\hline \multicolumn{12}{|l|}{ MRx (faixa etária) } \\
\hline 0 a 4 anos & 66 & 37 & 22 & 15 & 7 & 23 & 15 & 23 & 38 & 53 & 30 \\
\hline 5 a 19 anos & 38 & 26 & 30 & 33 & 35 & 31 & 34 & 32 & 57 & 44 & 36 \\
\hline 20 a 59 anos & 221 & 137 & 155 & 155 & 148 & 152 & 155 & 160 & 164 & 191 & 164 \\
\hline 60 anos ou mais & 214 & 151 & 134 & 181 & 207 & 215 & 215 & 173 & 239 & 247 & 198 \\
\hline Total & 162 & 103 & 112 & 119 & 119 & 124 & 126 & 125 & 143 & 159 & 129 \\
\hline \multicolumn{12}{|l|}{ MIP (faixa etária) } \\
\hline 0 a 4 anos & 7,4 & 14,8 & 0 & 0 & 0 & 7,7 & 0 & 0 & 0 & 0 & 3,0 \\
\hline 5 a 19 anos & 0 & 0 & 0 & 0 & 6,2 & 0 & 0 & 0 & 0 & 0 & 0,6 \\
\hline 20 a 59 anos & 5,6 & 3,7 & 5,4 & 1,8 & 0,9 & 2,7 & 0,9 & 0 & 3,4 & 0,8 & 2,5 \\
\hline 60 anos ou mais & 4,5 & 8,9 & 13,0 & 3,9 & 0 & 7,3 & 0 & 10,2 & 3,3 & 3,1 & 5,4 \\
\hline Total & 4,1 & 4,1 & 4,6 & 1,5 & 2,0 & 3,0 & 0,5 & 1,5 & 2,4 & 1,0 & 2,5 \\
\hline
\end{tabular}

${ }^{a}$ Incidência de hospitalização por 100 mil habitantes.

b Mortalidade por 100 milhões de habitantes.

Medicamentos isentos de prescrição (MIP); Medicamentos com prescrição (MRx). 
Tabela 3. Incidência de hospitalização e mortalidade por intoxicação medicamentosa segundo região, Brasil, 2009-2018.

\begin{tabular}{|c|c|c|c|c|c|c|c|c|c|c|c|}
\hline & \multicolumn{10}{|c|}{ Ano } & \multirow{2}{*}{$\begin{array}{l}\text { Média } \\
\text { anual }\end{array}$} \\
\hline & 2009 & 2010 & 2011 & 2012 & 2013 & 2014 & 2015 & 2016 & 2017 & 2018 & \\
\hline \multicolumn{12}{|l|}{ Hospitalização $^{a}$} \\
\hline \multicolumn{12}{|l|}{ MRx (por região) } \\
\hline Norte & 3,23 & 2,54 & 2,80 & 3,72 & 3,71 & 1,62 & 1,29 & 1,17 & 1,52 & 1,33 & 2,29 \\
\hline Centro-oeste & 9,28 & 5,91 & 5,34 & 5,17 & 4,81 & 3,84 & 2,99 & 3,49 & 3,94 & 3,63 & 4,84 \\
\hline Nordeste & 4,83 & 2,83 & 2,73 & 2,79 & 2,42 & 2,34 & 2,18 & 2,00 & 2,09 & 2,43 & 2,66 \\
\hline Sudeste & 6,64 & 5,09 & 5,08 & 4,88 & 4,85 & 4,94 & 4,38 & 4,61 & 5,18 & 5,76 & 5,14 \\
\hline Sul & 8,35 & 5,53 & 4,81 & 4,94 & 5,35 & 5,38 & 4,96 & 5,81 & 7,18 & 7,72 & 6,00 \\
\hline Brasil & 6,10 & 4,23 & 4,08 & 4,10 & 3,99 & 3,72 & 3,49 & 3,47 & 3,98 & 4,36 & 4,15 \\
\hline \multicolumn{12}{|l|}{ MIP (por região) } \\
\hline Norte & 0,13 & 0,05 & 0,06 & 0,10 & 0,11 & 0,12 & 0,09 & 0,02 & 0,08 & 0,04 & 0,08 \\
\hline Centro-oeste & 0,57 & 0,26 & 0,21 & 0,20 & 0,19 & 0,24 & 0,10 & 0,11 & 0,13 & 0,15 & 0,22 \\
\hline Nordeste & 0,17 & 0,11 & 0,15 & 0,10 & 0,11 & 0,08 & 0,09 & 0,07 & 0,05 & 0,06 & 0,10 \\
\hline Sudeste & 0,17 & 0,09 & 0,10 & 0,12 & 0,12 & 0,12 & 0,12 & 0,12 & 0,10 & 0,09 & 0,12 \\
\hline Sul & 0,27 & 0,17 & 0,15 & 0,21 & 0,17 & 0,18 & 0,21 & 0,19 & 0,20 & 0,21 & 0,20 \\
\hline Brasil & 0,21 & 0,12 & 0,13 & 0,13 & 0,14 & 0,13 & 0,12 & 0,11 & 0,11 & 0,11 & 0,13 \\
\hline \multicolumn{12}{|l|}{ Mortalidade $^{b}$} \\
\hline \multicolumn{12}{|l|}{ MRx (por região) } \\
\hline Norte & 68 & 80 & 58 & 74 & 67 & 49 & 53 & 48 & 128 & 53 & 68 \\
\hline Centro-oeste & 222 & 130 & 111 & 113 & 177 & 103 & 116 & 71 & 148 & 111 & 130 \\
\hline Nordeste & 224 & 76 & 105 & 131 & 120 & 103 & 91 & 135 & 101 & 147 & 123 \\
\hline Sudeste & 153 & 137 & 149 & 154 & 155 & 191 & 148 & 176 & 194 & 226 & 168 \\
\hline Sul & 205 & 136 & 123 & 124 & 132 & 157 & 134 & 139 & 236 & 249 & 164 \\
\hline Brasil & 158 & 101 & 108 & 115 & 115 & 120 & 121 & 120 & 139 & 155 & 125 \\
\hline \multicolumn{12}{|l|}{ MIP (por região) } \\
\hline Norte & 0 & 5,73 & 0 & 0 & 10,94 & 5,41 & 0 & 0 & 0 & 5,52 & 2,76 \\
\hline Centro-oeste & 7,07 & 0 & 25,42 & 0 & 0 & 6,60 & 0 & 0 & 0 & 0 & 3,91 \\
\hline Nordeste & 5,60 & 13,52 & 6,79 & 1,83 & 1,54 & 6,11 & 0 & 3,02 & 3,56 & 2,99 & 4,50 \\
\hline Sudeste & 5,70 & 1,22 & 2,05 & 2,03 & 0 & 1,18 & 1,08 & 1,96 & 1,15 & 0 & 1,64 \\
\hline Sul & 0 & 0 & 7,14 & 3,54 & 2,98 & 3,48 & 0 & 5,78 & 9,14 & 0 & 3,21 \\
\hline Brasil & 4,09 & 4,24 & 4,43 & 1,46 & 2,06 & 3,02 & 0,46 & 1,49 & 2,32 & 0,94 & 2,45 \\
\hline
\end{tabular}

a Incidência de hospitalização por 100 mil habitantes padronizada para a distribuição da população mundial (OMS, 2000-2025).

${ }^{\mathrm{b}}$ Mortalidade por 100 milhões de habitantes padronizada para a distribuição da população mundial (OMS, 2000-2025).

Medicamentos isentos de prescrição (MIP); Medicamentos com prescrição (MRx).

brasileira, são apresentados na Tabela 4. No período de 2009 a 2018, a tendência da incidência de hospitalizações por intoxicação medicamentosa por MRx foi estacionária, variando de ascendente na região sul a decrescente nas regiões centro-oeste e nordeste. Enquanto a incidência de internações por MIP teve tendência decrescente no Brasil, especialmente nas regiões centro-oeste e nordeste. A tendência da mortalidade das intoxicações por MRx foi ascendente no Brasil, inclusive nas regiões sul e sudeste. Já a mortalidade das intoxicações por MIP mostrou tendência decrescente no país. 
Tabela 4. Hospitalizações e óbitos, coeficiente de regressão e tendência de hospitalizações e mortalidade por intoxicação medicamentosa segundo região, Brasil, 2009-2018.

\begin{tabular}{|c|c|c|c|c|c|}
\hline & $\mathbf{n}$ & Coeficiente & IC $95 \%$ & $\mathbf{p}$ & Tendência \\
\hline \multicolumn{6}{|l|}{ Hospitalização } \\
\hline \multicolumn{6}{|l|}{ MRx (por região) } \\
\hline Norte & 3.868 & $-0,268$ & $-0,586$ a 0,050 & 0,087 & Estacionária \\
\hline Centro-oeste & 7.064 & $-0,224$ & $-0,407$ a $-0,041$ & 0,023 & Decrescente \\
\hline Nordeste & 14.375 & $-0,080$ & $-0,146$ a $-0,014$ & 0,024 & Decrescente \\
\hline Sudeste & 41.522 & 0,124 & $-0,020$ a 0,268 & 0,082 & Estacionária \\
\hline Sul & 16.446 & 0,514 & 0,328 a 0,700 & 0,000 & Ascendente \\
\hline Brasil & 83.275 & 0,049 & $-0,072$ a 0,169 & 0,371 & Estacionária \\
\hline \multicolumn{6}{|l|}{ MIP (por região) } \\
\hline Norte & 142 & $-0,002$ & $-0,0153$ a 0,010 & 0,605 & Estacionária \\
\hline Centro-oeste & 317 & $-0,014$ & $-0,026$ a $-0,002$ & 0,025 & Decrescente \\
\hline Nordeste & 545 & $-0,010$ & $-0,011$ a $-0,009$ & 0.000 & Decrescente \\
\hline Sudeste & 977 & $-0,000$ & $-0,003$ a 0,002 & 0,705 & Estacionária \\
\hline Sul & 555 & 0,004 & $-0,000$ a 0,009 & 0,052 & Estacionária \\
\hline Brasil & 2.536 & $-0,004$ & $-0,005$ a $-0,002$ & 0,001 & Decrescente \\
\hline \multicolumn{6}{|l|}{ Óbitos } \\
\hline \multicolumn{6}{|l|}{ MRx (por região) } \\
\hline Norte & 108 & 0,283 & $-5,478$ a 6,045 & 0,911 & Estacionária \\
\hline Centro-oeste & 172 & $-3,446$ & $-9,001$ a 2,109 & 0,186 & Estacionária \\
\hline Nordeste & 621 & 1,772 & $-1,879$ a 5,423 & 0,289 & Estacionária \\
\hline Sudeste & 1.278 & 6,680 & 3,959 a 9,401 & 0,001 & Ascendente \\
\hline Sul & 416 & 15,527 & 6,757 a 24,297 & 0,004 & Ascendente \\
\hline Brasil & 2.595 & 5,885 & 3,635 a 8,136 & 0,000 & Ascendente \\
\hline \multicolumn{6}{|l|}{ MIP (por região) } \\
\hline Norte & 108 & 0,283 & $-5,478$ a 6,045 & 0,911 & Estacionária \\
\hline Centro-oeste & 172 & $-3,446$ & $-9,001$ a 2,109 & 0,186 & Estacionária \\
\hline Nordeste & 621 & 1,772 & $-1,879$ a 5,423 & 0,289 & Estacionária \\
\hline Sudeste & 1.278 & 6,680 & 3,959 a 9,401 & 0,001 & Ascendente \\
\hline Sul & 416 & 15,527 & 6,757 a 24,297 & 0,004 & Ascendente \\
\hline Brasil & 2.595 & 5,885 & 3,635 a 8,136 & 0,000 & Ascendente \\
\hline
\end{tabular}

Medicamentos isentos de prescrição (MIP); Medicamentos com prescrição (MRx).

\section{DISCUSSÃO}

No decênio avaliado neste estudo (2009-2018), a maioria das internações por intoxicação medicamentosa foi causada por MRx (97\%), uma incidência 32 vezes maior que a de hospitalizações por MIP. Apesar dessa grande diferença, indicando o papel preponderante das MRx comparadas às MIP nos casos graves, os dados de comercialização durante a segunda metade da década estudada (2014-2018) mostram que foram vendidas menos doses de MRx (7,2 bilhões) do que de MIP (7,7 bilhões) $)^{15}$. A isenção de prescrição baseia-se sobretudo no baixo potencial de toxicidade desses produtos, que têm reações adversas reversíveis após suspensão de uso e reações graves apenas com a administração de grande quantidade do produto, além de apresentarem janela terapêutica segura ${ }^{16}$. Possivelmente, este perfil de segurança é responsável pelo menor número de intoxicações graves e hospitalizações causadas por MIP. A mortalidade muito maior observada nas internações por MRx, cerca de 50 vezes maior, sustenta essa argumentação. 
A ANVISA é responsável pela regulamentação de propaganda, publicidade, informação e outras práticas com objetivo de divulgação ou promoção comercial de medicamentos ${ }^{17}$, incluindo também os materiais elaborados pela indústria farmacêutica com a finalidade de apresentar aos profissionais prescritores e dispensadores. Os resultados desta pesquisa, indicando mortalidade muito mais elevada no uso de medicamentos com prescrição, têm implicações importantes, que justificam o aprimoramento dessa regulamentação para prevenir a automedicação e o uso de medicamentos de forma não racional.

As internações foram mais comuns no sexo feminino, enquanto a mortalidade foi maior no masculino, tanto nas intoxicações por MIP como por MRx. Mota et al. (2012), analisando o decênio de 1996 a 2005, também observaram maior prevalência de óbitos por intoxicações medicamentosas em homens ${ }^{18}$. As internações por intoxicação medicamentosa foram mais frequentes em menores de 5 anos de idade, independentemente do tipo de medicação. A ingestão acidental de medicamentos é mais comum nessa faixa etária ${ }^{19,20}$, além disso, o menor peso corporal nesse grupo torna-os mais vulneráveis à intoxicação com doses relativamente menores ${ }^{21,22}$. Quanto às taxas de mortalidade, elas foram maiores nos mais idosos, semelhante ao observado em outros estudos ${ }^{23,24}$. É possível que o uso mais frequente de medicamentos e a susceptibilidade aumentada à toxicidade, associados à diminuição da capacidade de metabolização e excreção de medicamentos, decorrente do avanço da idade, possam ter contribuído para maior mortalidade observada em idosos ${ }^{24,25}$.

A incidência das internações por MIP e MRx variou no Brasil, onde as regiões sul, centro-oeste e sudeste apresentaram as maiores taxas e as regiões norte e nordeste as menores. A mortalidade das intoxicações variou segundo a região do país, no sudeste e sul ocorreram as maiores taxas de mortalidade por MRx, enquanto as regiões nordeste e centro-oeste apresentaram a maior mortalidade por MIP. Existem evidências no Brasil da associação entre maior poder aquisitivo e maior utilização de medicamentos ${ }^{26}$, essa pode ser uma explicação para taxas mais elevadas e crescentes de intoxicação medicamentosa nas regiões sul e sudeste onde a renda per capita da população é maior do que as demais regiões do país ${ }^{27}$. Desse modo, as diferenças observadas podem ser desdobramentos das desigualdades na disponibilidade e no acesso a medicamentos das populações residentes nessas regiões. Podem ser também consequências de diferenças nas coberturas dos serviços de saúde responsáveis pela notificação dos casos e dos óbitos por intoxicações medicamentosas nas diferentes regiões no Brasil.

A tendência da incidência das internações por MRx foi estacionária, mas a mortalidade aumentou durante o decênio, enquanto houve tendência decrescente na mortalidade e na incidência de internações por MIP no mesmo período. O perfil epidemiológico da população brasileira vem se modificando com aumento da prevalência de doenças crônicas e consequente uso de medicamentos com maior frequência ${ }^{28,29}$. A polifarmácia tem se tornado um problema entre idosos, no Brasil, estima-se que $82 \%$ das pessoas acima de 60 anos façam uso de ao menos um medicamento ${ }^{30}$. Embora esses medicamentos possam aumentar a qualidade e a expectativa de vida, eles também podem trazer riscos a essa população devido às interações medicamentosas e à alteração da fisiologia, causada pela senescência ${ }^{31}$. Isto pode ser um dos fatores responsáveis pela manutenção da incidência das internações e pelo aumento da mortalidade nos casos de intoxicação por MRx.

\section{Limitações e Méritos}

A natureza retrospectiva desse estudo baseado em banco de dados preexistente, em que as informações disponíveis são limitadas, impediu que algumas características relativas às circunstâncias, às causas e ao tratamento das internações por intoxicação medicamentosa pudessem ser investigadas. Além disso, as informações referem-se apenas aos serviços de saúde públicos, não abrangendo os serviços privados, portanto, as taxas produzidas estão subestimadas. No entanto, a cobertura nacional dos dados, incluindo um período de vários anos, permitiu comparações entre diferentes regiões e forneceu 
medidas de tendência para a incidência das internações por intoxicação medicamentosa, assim como para a mortalidade.

\section{CONCLUSÕES}

As internações por intoxicação medicamentosa têm grande impacto e importância na saúde pública, merecendo atenção especialmente por sua natureza potencialmente prevenível. Apesar do maior acesso a medicamentos com isenção de prescrição, a quase totalidade das hospitalizações e dos óbitos no Brasil entre 2009 e 2018 ocorreu em decorrência de intoxicações causadas por medicamentos com prescrição obrigatória. Nossos resultados reforçam a necessidade de estudos analíticos para identificar os determinantes e prevenir a ocorrência das intoxicações medicamentosas.

\section{REFERÊNCIAS}

1. Agência Nacional de Vigilância Sanitária. Resolução No 4, de 10 de fevereiro de 2009. Dispõe sobre as normas de farmacovigilância para os detentores de registro de medicamentos de uso humano. Brasília, DF: Anvisa; 2009 [citado 16 mar 2021]. Disponível em: https://bvsms.saude.gov.br/bvs/saudelegis/anvisa/2009/res0004_10_02_2009.html

2. Amaral DA. Intoxicações por medicamento. In: Seizi O, organizador. Fundamentos de toxicologia. 2.ed. São Paulo: Varella; 2003. p.367-79.

3. World Health Organization (WHO). Guidelines on the prevention of toxic exposures. Education and public awareness activities / International Programme on Chemical Safety.2004 Disponível em: https://www.who.int/ipcs/features/prevention_guidelines.pdf4.

4. Sistema Nacional de Informações Tóxico-Farmacológicas: dados de intoxicação: dados nacionais. Rio de Janeiro: Fiocruz; c2009 [citado 16 mar 2021]. Disponível em: https://sinitox.icict.fiocruz.br/dados-nacionais

5. National Poisons Information Service (NPIS). National Poisons Information Service Report 2018/19. Disponível em: https://www.npis.org/Download/NPISAnnualReport2018-19.pdf6.

6. Gummin DD, Mowry JB, Spyker DA, Brooks DE, Beuhler MC, Rivers LJ, et al. 2018 Annual Report of the American Association of Poison Control Centers' National Poison Data System (NPDS): 36th Annual Report. Clin Toxicol (Phila). 2019;57(12):1220-413. https://doi.org/10.1080/15563650.2019.1677022

7. Richardson R, Charters T, King N, Harper S. Trends in educational inequalities in drug poisoning mortality: United States, 1994-2010. Am J Public Health. 2015;105(9):1859-65. https://doi.org/10.2105/AJPH.2015.302697

8. Brasil. Lei № 5991, de 17 de dezembro de 1973. Dispõe sobre o Controle Sanitário do Comércio de Drogas, Medicamentos, Insumos Farmacêuticos e Correlatos, e dá outras Providências. Brasíla, DF; 1973 [citado 16 mar 2021]. Disponível em: http://www.planalto.gov.br/ccivil_03/leis/l5991.htm

9. Agência Nacional de Vigilância Sanitária. Instrução Normativa $N^{0} 11$, de 29 de setembro de 2016. Dispõe sobre a lista de medicamentos isentos de prescrição. Diário Oficial da União. 30 set 2016 [citado 28 dez 2020]; Seção 1:99. Disponível em: https://www.in.gov.br/materia/-/ asset_publisher/Kujrw0TZC2Mb/content/id/23376708/do1-2016-08-03-resolucao-rdc-n-98-de1-de-agosto-de-2016-23376586

10. Instituto Brasileiro de Geografia e Estatística. Estimativas da População. Rio de Janeiro: IBGE; 2020 [citado 28 dez 2020]. Disponível em: https://www.ibge.gov.br/estatisticas/sociais/ populacao.html

11. Ahmad OB, Boschi-Pinto C, Lopez AD, Murray CJL, Lozano R, Inoue M. Age standardization of rates: a new WHO standard. Geneva (CH): WHO; 2001 [citado 16 mar 2021]. (GPE Discussion Paper Series; $\left.n^{\circ} 31\right)$. Disponível em: http://www.who.int/healthinfo/paper31.pdf

12. Becketti S. Prais-Winsten regression. Stata Tech Bull. 1995 [citado 16 mar 2021];(25):26-9. Disponível em: https://www.stata-press.com/journals/stbcontents/stb25.pdf

13. Park RE, Mitchell BM. Estimating the autocorrelated error model with trended data. J Econom. 1980;13(2):185-201. 
14. Gaynor PE, Kirkpatrick RC. Introduction to time-series modeling and forecasting in business and economics. New York: McGraw-Hill; 1993. (McGraw-Hill economic series); (Wiley series in probability and mathematical statistics).

15. Pharma, Sales and Marketing Terms - IMS Health - PMB - Pharmaceutical Market Brasil. [cited 30 Jul 2021]. Available: https://brazilsfe.blogspot.com/2015/12/pharma-terms-ims-o-quee-pmb-pharmaceutical-market-brasil.html

16. Agência Nacional de Vigilância Sanitária. Resolução - RDC Nº 98 de $1^{\circ}$ de agosto de 2016. Dispõe sobre os critérios e procedimentos para o enquadramento de medicamentos como isentos de prescrição e o reenquadramento como medicamentos sob prescrição, e dá outras providências. Diário Oficial da União. 3 ago 2016 [citado 16 mar 2021]; Seção 1:32. Disponível em: https://www.in.gov.br/materia/-/asset_ publisher/Kujrw0TZC2Mb/content/id/23376708/do1-2016-08-03-resolucao-rdc-n-98-de-1-deagosto-de-2016-23376586

17. Agência Nacional de Vigilância Sanitária. Resolução-RDC No 96, de 17 de dezembro de 2008. Dispõe sobre a propaganda, publicidade, informação e outras práticas cujo objetivo seja a divulgação ou promoção comercial de medicamentos. Brasília, DF: Anvisa; 2008 [citado 16 mar 2021]. Disponível em: http://bvsms.saude.gov.br/bvs/saudelegis/anvisa/2008/ rdc0096_17_12_2008.html

18. Mota DM, Melo JRR, Freitas DRC, Machado M. Perfil da mortalidade por intoxicação com medicamentos no Brasil, 1996-2005: retrato de uma década. Cienc Saude Coletiva. 2012;17(1):61-70. https://doi.org/10.1590/S1413-8123201200010000

19. Lee J, Fan NC, Yao TC, Hsia SH, Lee EP, Huang JL, et al. Clinical spectrum of acute poisoning in children admitted to the pediatric emergency department. Pediatr Neonatol. 2019;60(1):59-67. https://doi.org/10.1016/j.pedneo.2018.04.001

20. Lessa MA, Bochner R. Analysis of hospitalizations of children under one year of age due to drug intoxication and adverse events in Brazil. Rev Bras Epidemiol. 2008;11(4):1-15. https://doi.org/10.1590/S1415-790X2008000400013

21. Lacroix J, Gaudreault P, Gauthier M. Admission to a pediatric intensive care unit for poisoning: a review of 105 cases. Crit Care Med. 1989;17(8):748-50. https://doi.org/10.1097/00003246-198908000-00005

22. Toce MS, Burns MM. The poisoned pediatric patient. Pediatr Rev. 2017;38(5):207-20. https://doi.org/10.1542/pir.2016-0130

23. Santos GAS, Boing AC. Mortalidade e internações hospitalares por intoxicações e reações adversas a medicamentos no Brasil: análise de 2000 a 2014. Cad Saude Publica. 2018;34(6):e00100917. https://doi.org/10.1590/0102-311X00100917

24. Ramos LR, Tavares NUL, Bertoldi AD, Farias MR, Oliveira MA, Luiza VL, et al. Polypharmacy and polymorbidity in older adults in Brazil: a public health challenge. Rev Saude Publica. 2016;50 Suppl 2:1s-12s. https://doi.org/10.1590/S1518-8787.2016050006145

25. Baldoni AO, Chequer FMD, Ferraz ERA Oliveira DP, Pereira LRL, Dorta DJ. Elderly and drugs: risks and necessity of rational use. Braz J Pharm Sci. 2010;46(4):617-32. https://doi.org/10.1590/S1984-82502010000400003

26. Bertoldi AD, Dal Pizzol TS, Ramos LR, Mengue SS, Luiza VL, Tavares NUL, et al. Sociodemographic profile of medicines users in Brazil: results from the 2014 PNAUM survey. Rev Saude Publica. 2016;50 Suppl 2:1s-10s. https://doi.org/10.1590/S1518-8787.2016050006119

27. Agência IBGE Notícias. IBGE divulga o rendimento per capita 2017. Rio de Janeiro: IBGE; 2018 [citado 16 mar 2021]. Disponível em: https://agenciadenoticias.ibge.gov.br/agencia-salade-imprensa/2013-agencia-de-noticias/releases/20154-ibge-divulga-o-rendimento-domiciliarper-capita-2017

28. Schramm JMA, Oliveira AF, Leite IC, Valente JG, Gadelha AMJ, Portela MC, et al. Transição epidemiológica e o estudo de carga de doença no Brasil. Cienc Saude Coletiva. 2004;9(4):897-908. https://doi.org/10.1590/S1413-81232004000400011

29. Marinho F, Passos VMA, França EB. Novo século, novos desafios: mudança no perfil da carga de doença no Brasil de 1990 a 2010. Epidemiol Serv Saude. 2016;25(4):713-24. https://doi.org/10.5123/S1679-49742016000400005

30. Bezerra TA, Brito MAA, Costa KNFM. Characterization of medication use among elderly people attended at a family health care service. Cogitare Enferm. 2016;21(1):1-10. 
31. Pagno AR, Gross CB, Gewehr DM, Colet CF, Berlezi EM.

A terapêutica medicamentosa, interações potenciais e iatrogenia como fatores relacionados à fragilidade em idosos. Rev Bras Geriatr Gerontol. 2018;21(5):610-9. https://doi.org/10.1590/1981-22562018021.180085

Financiamento: Esse protocolo de estudo foi desenvolvido de forma independente pelos investigadores e contou com apoio financeiro incondicional da Associação Brasileira da Indústria de Medicamentos Isentos de Prescrição (ABIMIP).

Contribuição dos Autores: Concepção e planejamento do estudo: FGD, MNP, NAV, MCCA, EDMJ. Coleta, análise e interpretação dos dados: FGD, MNP, NAV, MCCA, EDMJ. Elaboração ou revisão do manuscrito: FGD, MNP, NAV, MCCA, EDMJ. Aprovação da versão final: FGD, MNP, NAV, MCCA, EDMJ. Responsabilidade pública pelo conteúdo do artigo: FGD, MNP, NAV, MCCA, EDMJ.

Conflito de Interesses: Os autores declaram não haver conflito de interesses. 